\title{
Authenticated Dictionary Based on Frequency
}

\author{
Kévin Atighehchi, Alexis Bonnecaze, and Traian Muntean \\ Aix Marseille University, CNRS, Centrale Marseille, ERISCS, I2M, UMR 7373, \\ 13453 Marseille, France \\ \{firstname. lastname\}@univ-amu.fr
}

\begin{abstract}
We propose a model for data authentication which takes into account the behavior of the clients who perform queries. Our model reduces the size of the authenticated proof when the frequency of the query corresponding to a given data is higher. Existing models implicitly assume the frequency distribution of queries to be uniform, but in reality, this distribution generally follows Zipf's law. Therefore, our model better reflects reality and the communication cost between clients and the server provider is reduced allowing the server to save bandwith. When the frequency distribution follows Zipf's law, we obtain a gain of at least $20 \%$ on the average proof size compared to existing schemes.
\end{abstract}

Keywords: Authenticated dictionary, Data structure, Merkle tree, Zipf.

\section{Introduction}

Authenticated dictionaries are used to organize and manage a collection of data in order to answer queries on these data and to certify the answers. They have been heavily studied recently and have many applications including certificate revocation in public key infrastructure [47|10 16], geographic information system querying, or third party data publication on the Internet [5]2]. This last application is of great interest with the advent of cloud computing and Web services. For example, it is important that a user who consults a Web page can be confident of the authenticity of that page (or some of its contents).

Classical schemes involve three actors [22 89]: a trusted source which is generally the owner of the data, an untrusted provider also called directory and a set of users (also called clients). The directory receives a set of data from the source together with authentication information. These contents are stored by both the source and the directory but only the latter communicates with users. Therefore, as shown in Figure 1 , users communicate directly with the directory to query the authentication information on a given data. This information contains a cryptographic proof and allows the users to authenticate the data.

Most of authenticated dictionaries use Merkle trees, red-black trees or skip-lists as data structures. These structures are closely equivalent in terms of cost of storage, communication and time [22]. They are well adapted as long as no distinction is made between data. However, in some situations, it may be useful to manage data as a function of some parameters. In the case of publications on the Internet, some pages are 


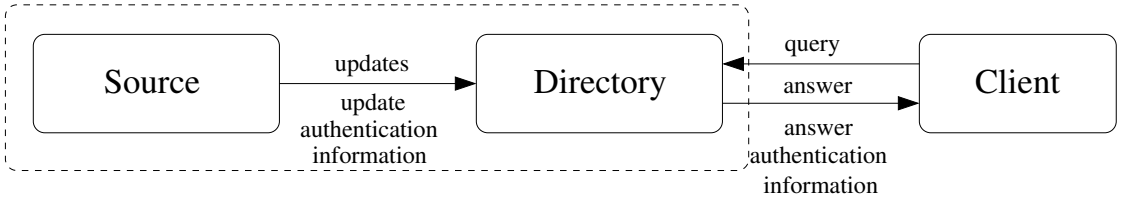

Fig. 1. The three-party authentication model

accessed more frequently, depending on user behavior. Some pages have a better reputation than others, and it may prove useful to order them following this criterion. in fact, any behavioural criterion could be taken into account.

In this paper, we introduce an authenticated dictionary scheme which takes into account the frequency of data being accessed. As regards Web traffic, it is well known that its frequency distribution follows Zipf's law [1|18|17|126|21]. More precisely, most traffic follows this law except for the traffic residue corresponding to very low frequencies. In fact, there is a drooping tail, which means that for these frequencies, the distribution decreases much faster than Zipf's law.

The paper is organized as follows. Section 2 contains background information regarding data structures and the dictionary problem. Section 3 introduces our scheme. We present the underlying data structures and the updating, searching and certification operations provided by the dictionary. In Section 4 we discuss the efficiency of our method, and show that, compared to existing schemes and when the frequency distribution follows Zipf's law, the reduction of proof size is better than $20 \%$.

\section{Background}

\subsection{Data Structures and Authentication}

Data structures represent a way of storing and organizing data so that searching, adding or deleting operations can be done efficiently. A static structure has a size that cannot be changed and therefore it is not possible to delete or add any data a posteriori. However, the size of dynamic data structures can change allowing insertion and deletion operations. In this paper, the term dynamic data structure refers to any data structure which accepts insertion and deletion of data at any position. The term append/disjoinonly data structure refers to any data structure which accepts insertion and deletion at the end of the structure. Examples of dynamic structures [13] are hash tables, trees like 2-3 Trees, B-Trees or red-black trees, or other random structures like non-deterministic skip-lists [20].

In addition to these basic features, data structures can be used to construct authenticating mechanisms. Data structures based on rooted graphs are well adapted to deal with such mechanisms [22]8] since authenticating all the data covered by the graph just requires one single signature and some hash computations. An example of authenticated data structure is the static Merkle tree [1415] of which the number of leaves is a power of 2. There exist variants accepting any number of leaves. Although these variants can still be considered as static, they can also be considered as append/disjoin-only data 
structures since structural changes can be done at the right side of the tree. This type of structure is suitable for time stamping [193]. In the following, we briefly detail one of these variants [19]. It is an almost balanced tree in which values of the internal nodes are calculated in the following way. Let $\left(e_{1}, e_{2}, \ldots, e_{n}\right)$ be the values of the leaves at the base of the tree. Values of nodes at the previous level are $\left(h\left(e_{2 i+1}, e_{2 i+2}\right)_{i=0 \ldots(n-2) / 2}\right)$ if $n$ is even, and $\left(h\left(e_{2 i+1}, e_{2 i+2}\right)_{i=0 \ldots(n-3) / 2}, e_{n}\right)$ otherwise. This process is repeated until a single value is obtained (this is the root node value). Adding an element $e^{*}$ after $e_{n}$ is a very simple operation. The value $v$ of the root of the smallest (perfectly) balanced subtree to where $e_{n}$ belongs is changed to $v^{\prime}=h\left(v, e^{*}\right)$. Then, values of the internal nodes on the path from this root to the root of the tree are updated. The disjoin operation is just the inverse operation. Note that this structure is equivalent to a deterministic skip-list. Finally, one might add that static structures should always be preferred for their better complexity when there is no need for complex operations.

\subsection{The Dictionary Problem}

The authenticated dictionary problem has already been defined in the literature, for example in [22 8]. In this section, we summarize the main features of an authenticated dictionary. The source has a set $S$ of elements which evolves over time through insertion and deletion of items. The directory maintains a copy of this set and its role is to answer queries from the users. A user may request a given element or may perform a membership query on $S$ in order to know whether an item belongs or not to $S$. The user must be able to verify the attached cryptographic proof (in particular, public information about the source must be available).

Efficiency makes the difference between a good dictionary and a bad one. This efficiency can be measured in terms of computation cost, which is the time taken by the computation together with the cost of the hardware (memory space and bandwidth) used by the entities. The size of the proofs is perhaps the most important parameter since it plays a significant role on the interface bandwidth of the directory. Moreover, it may reduce the time for a user to verify the answer to a query. The time spent by the directory to answer a query is also an important parameter when the number of users is very large. Space used by the data structure as well as source to directory communication should be optimized. Finally, the time to perform an update should also be optimized.

In this paper, our objective is to reduce the average size of the proof. This improvement is done at the expense of a slightly greater need for memory and computation of both the source and directory.

\section{A New Authenticated Dictionary Based on Frequency}

So far, authentication schemes have relied on data structures like Merkle trees or skiplists. These data structures allow us to obtain small sizes of proof. In this sense, they seem to be optimal whenever each data has the same probability to be queried. However, in real life, users can make more queries on a given data than another. This means that the frequency of queries may be far from uniform. In the case of publication on the Internet, some Web pages are consulted more frequently than others. Taking into 
account this parameter, we introduce a scheme in which the size of authentication proof answering a query is smaller when the frequency of this query is higher. We obtain the following benefits:

- for the directory, we minimize on average the LAN/WAN interface bandwidth usage. This interface bandwidth represents a critical aspect because the number of simultaneous queries may be high.

- If the directory caches proofs which are frequently queried, the number of proofs being cached will be higher, improving at the same time efficiency.

- On average, for a given user, the LAN/WAN interface bandwidth and the number of calculations to verify a proof is reduced.

When the frequency distribution is uniform, it is preferable to use an almost balanced tree (or an equivalent data structure). However, when the frequency distribution is not uniform, there is no reason to use such a data structure. Rather, we should look for unbalanced tree structures in order to improve efficiency, in particular on the size and construction of proofs.

\section{Zipf's law for an exponent $s=1$ (linear scale)}

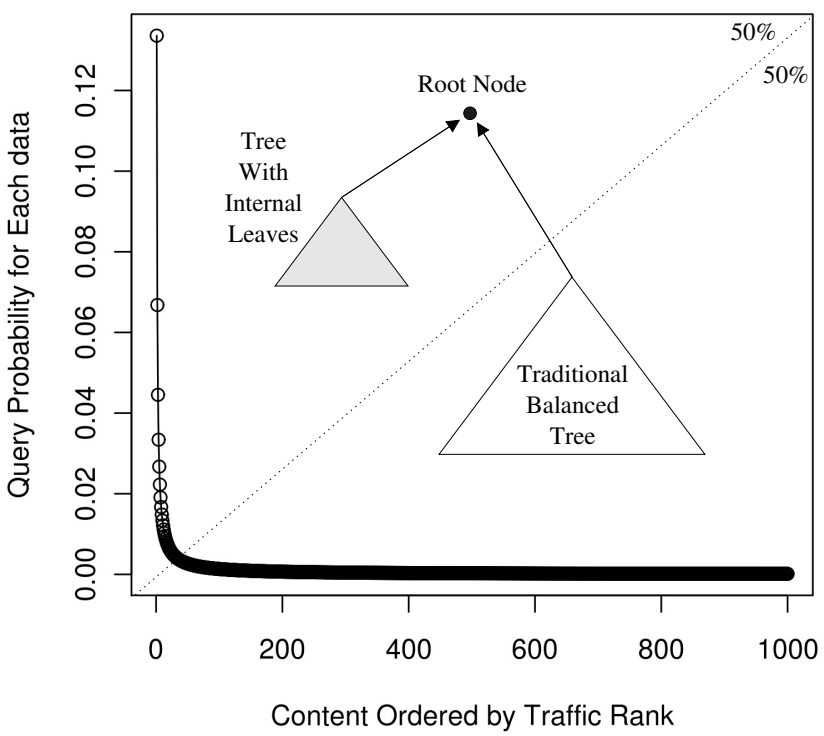

Fig. 2. Zipf's law and the tree $T$

We consider here a distribution which follows Zipf's law. Figure 2 shows that the distribution curve is close to the vertical axis for high frequency events whereas it is close to the horizontal axis for the many very low frequency events. The latter part of the curve (which corresponds to the lower tail) behaves like a uniform distribution. Therefore, if we had to construct an authenticated dictionary corresponding to the lower tail, we would certainly use a balanced tree or any equivalent data structure (denoted 
$T_{2}$ ). However, for the rest of the distribution, we should use an unbalanced tree (denoted $T_{1}$ ), having its leaves ever closer to the root as frequency increases. Finally, in order to take into account the whole distribution, we propose to use a tree $T$ whose root has $T_{1}$ as left child and $T_{2}$ as right child.

Since $T$ does not arrange data in order of key identifiers but in descending order of frequencies, we need to use two other (non authenticated) structures, one ordering all the data according to frequencies and the other one ordering data according to key identifiers. Our scheme relies on the following data structures.

- We assume the use of two efficient dynamic binary trees which serve to organize and manage data. The first one, denoted $A_{1}$, ranks the $\left(u_{i}\right)_{i=1 \ldots n}$ in ascending order and allows us to search a given $u_{i}$ and to retrieve its corresponding frequency. The searching operation only uses $A_{1}$ and is done in $\mathrm{O}(\log (n))$. The second one, denoted $A_{2}$, is used to arrange frequencies in decreasing order and allows the rank of a given frequency to be retrieved.

- Authentication proofs are constructed using the third data structure, $T$. Its right child $T_{2}$, is a Merkle-like tree which processes data having very low frequencies. The left child, $T_{1}$ is a height-balanced tree with special properties: each node has three children, two of them being either parent nodes or leaves and the third one being exclusively a leaf. The structure $T_{1}$ is designed to reduce the size of proofs corresponding to high frequency data. The place of each leaf depends on the frequency of the data. The higher the frequency, the closer the leaf is to the root.

Even though the system uses more data structures than existing authenticated dictionaries, the global memory space taken by these structures is not significantly increased. In fact, adding structures is mainly equivalent to adding pointers which do not have a high memory cost.

Remark 1. For the sake of simplicity we assume that all the frequencies are distinct. We note that this is in fact the case if we consider the exact Zipf distribution. Furthermore, for the construction of the structure, we just consider absolute frequencies (an absolute frequency being the number of data access requests).

The next subsection presents some details about this authenticated data structure and assumes the use of $A_{1}$ and $A_{2}$.

\subsection{Authenticated Data Structure Construction}

Let $n$ be the number of data. Considering the use of a cryptographic hash function $H$, let $\left\{u_{1}, u_{2}, \ldots, u_{n}\right\}$ be the set of hashed identifiers, let $\left\{c_{1}, c_{2}, \ldots, c_{n}\right\}$ be the set of hashed data and let $\left(f_{1}, f_{2}, \ldots, f_{n}\right)$ be the corresponding list of $n$ frequencies. We denote by $\Pi$ the permutation in $[1, \ldots, n+1]$ such that $u_{i}$ has a frequency $f_{\Pi(i)}$.

In order to construct our tree $T$ and its two children $T_{1}$ and $T_{2}$, we divide the data into two sets according to their frequencies, or more precisely in our case, according to the median of the frequency distribution. Each data corresponds to a leaf. Leaves of $T_{1}$ correspond to data having the highest frequencies $f_{1}, f_{2}, \ldots, f_{k}$ (ranked in descending order, where $k$ is the smallest integer such that $\sum_{i=1}^{k} f_{i} \geq\left(\sum_{i=1}^{n} f_{i}\right) / 2$. Leaves of $T_{2}$ 


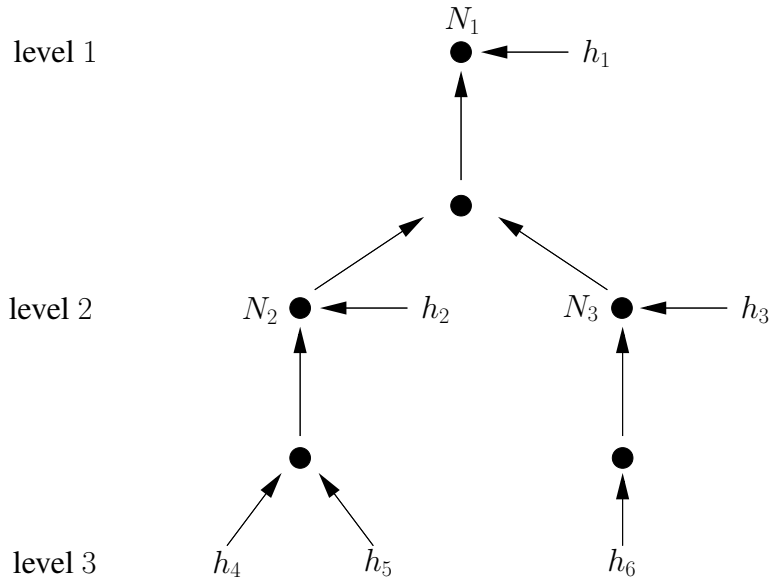

Fig. 3. Example of a tree $T_{1}$ for 6 elements. This diagram represents the flow of the computation of the nodes. Note that here the pairwise chaining for the computation of leaves is not depicted and that arrows denote the flow of information, not pointer values in the data structure.

correspond to the rest of the data. In practice, the number of leaves of $T_{2}$, denoted $N_{r}$, is much larger than that of $T_{1}$, denoted $N_{l}$. The tree $T_{2}$ is a Merkle tree for standard authenticated dictionaries. We also consider two special data, $\pm \infty$ both of frequency equal to zero which are used as sentinels in order to chain data according to their identity. The sentinel $+\infty$ is the last element of both lists and $f_{\Pi(n+1)}=f_{n+1}=0$.

The source constructs the ordered sets

$$
L^{u}=\left\{\left(u_{1}, f_{\Pi(1)}\right), \ldots,\left(u_{n}, f_{\Pi(n)}\right),(+\infty, 0)\right\}
$$

and

$$
L^{f}=\left\{\left(u_{\Pi^{-1}(1)}, f_{1}\right), \ldots,\left(u_{\Pi^{-1}(n)}, f_{n}\right),\left(u_{\Pi^{-1}(n)}, f_{n}\right),(+\infty, 0)\right\} .
$$

In the first list the values $u_{i}$ are ordered from the smallest to the largest, whereas the second list is ranked according to frequency. From these lists, the source calculates the tree $T$. Calculation of a leaf $h_{i}$ is done as follows:

- $h_{\Pi(1)}=H\left(-\infty, u_{1}, c_{1}\right)$,

- $h_{\Pi(i)}=H\left(u_{i-1}, u_{i}, c_{i}\right)$ where $i \in[2, \ldots, n]$,

- $h_{\Pi(+\infty)}=H\left(u_{n},+\infty, 0\right)$,

where 0 denotes empty content. Note that a pairwise chaining between the $u_{i}$ (and $-\infty$, $+\infty$ ) is used when calculating the leaves, this device serves for constructing proofs of non-existence.

We determine $i$ such that $2^{i} \leq N_{l}<2^{i+1}-1$. The calculation of nodes of $T_{1}$ is done as follows:

- $N_{k}=H\left(h_{k}, H\left(h_{2 k}, h_{2 k+1}\right)\right)$ for $k \in \llbracket 2^{i-1},\left\lfloor\frac{N_{l}}{2}\right\rfloor \llbracket$;

- $N_{\left\lfloor\frac{N_{l}}{2}\right\rfloor}=H\left(h_{\left\lfloor\frac{N_{l}}{2}\right\rfloor}, H\left(h_{2\left\lfloor\frac{N_{l}}{2}\right\rfloor}, h_{2\left\lfloor\frac{N_{l}}{2}\right\rfloor+1}\right)\right)$ if $N_{l}$ is odd,

$N_{\left\lfloor\frac{N_{l}}{2}\right\rfloor}=H\left(h_{\left\lfloor\frac{N_{l}}{2}\right\rfloor}, H\left(h_{2\left\lfloor\frac{N_{l}}{2}\right\rfloor}\right)\right)$ otherwise; 


$$
\begin{aligned}
& \text { - } N_{k}=H\left(h_{k}, H\left(N_{2 k}, N_{2 k+1}\right)\right) \text { for } k \in \llbracket 2^{i-2},\left\lfloor\frac{N_{l}}{4}\right\rfloor \llbracket \text {; } \\
& \text { - } N_{\left\lfloor\frac{N_{l}}{4}\right\rfloor}=H\left(h_{\left\lfloor\frac{N_{l}}{4}\right\rfloor}, H\left(N_{2\left\lfloor\frac{N_{l}}{4}\right\rfloor}, h_{2\left\lfloor\frac{N_{l}}{4}\right\rfloor+1}\right)\right) \text { if }\left\lfloor\frac{N_{l}}{2}\right\rfloor \text { is even, } \\
& H\left(h_{\left\lfloor\frac{N_{l}}{4}\right\rfloor}, H\left(h_{2\left\lfloor\frac{N_{l}}{4}\right\rfloor}, h_{2\left\lfloor\frac{N_{l}}{4}\right\rfloor+1}\right)\right) \text { otherwise; } \\
& \text { - } N_{k}=H\left(h_{k}, H\left(h_{2 k}, h_{2 k+1}\right)\right) \text { for } k \in \rrbracket\left\lfloor\frac{N_{l}}{4}\right\rfloor, 2^{i-1}-1 \rrbracket \text {; } \\
& \text { - } N_{k}=H\left(h_{k}, H\left(N_{2 k}, N_{2 k+1}\right)\right) \text { for } k \in \llbracket 2^{j}, 2^{j+1}-1 \rrbracket \text { and } j \in \llbracket 0, i-3 \rrbracket \text {; }
\end{aligned}
$$

Leaves are listed in descending order of frequency, from the root to the base level and in a given level from left to right. Figure 3 shows the structure of $T_{1}$ for 6 data.

The calculation of nodes of $T_{2}$ is not detailed since $T_{2}$ is a Merkle-like tree. When the tree $T$ is calculated, the source transmits the list of elements $\left(I d_{i}, c_{i}\right)_{i=1 \ldots n}$ together with the timestamped signature of the root node of $T$ to the directory. Then, the directory is able to construct the data structures.

\subsection{Proof Construction and Verification Algorithms}

In order to construct a proof of existence or non-existence for a data of (hashed) identifier $u$, we first use $A_{1}$ to determine the frequency $f_{\pi(j)}$ which corresponds to the smallest $u_{j}$ such that $u_{j} \geq u$. Then, we use $A_{2}$ to obtain the place $\pi(j)$ of this frequency. Suppose that $h_{\pi(j)}$ is a leaf of $T_{1}$. The binary representation of $\pi(j)$ is used to obtain the correct path in $T_{1}$ leading to the authentication proof. We consider a list $P$ initially empty, which will contain the hashed values representing the proof. When we know the path, the construction of the proof is similar to the one used in a Merkle tree:

- The value of the root node of $T_{2}$ is added to $P$.

- The most significant bit of $\pi(j)$ is not considered but we consider the following one. If this bit is equal to 0 , we add to $P$ both the value of the right child node and of the internal leaf and we move to the left node. If this bit is equal to 1 , we add to $P$ both the value of the left child node and of the internal leaf and we move to the right node.

- The process is repeated for the next bit and so forth until the last bit of $\pi(j)$. Note that, at the end, if the data corresponds to an internal leaf, we add to $P$ the hash of the concatenation of the two children (or the hash of the child, if there is just one child).

- In the case of a proof of existence, we add to $P$ the value $u_{j-1}$. In the case of a proof of non existence, we add to $P$ the values $u_{j-1}, u_{j}$ and $c_{j}$.

All the other cases (and in particular the one where $h_{\pi(j)}$ belongs to $T_{2}$ ) can be easily handled and are left to the reader. The verification of the proof is done by the user and involves recalculating the root node of $T$ from the value of the leaf corresponding to the data and hashes of the values of the proof. Note that the use of a commutative hash [8] for node calculation facilitates the calculation of the verification and slightly reduces the size of the proof.

\subsection{Updating Algorithms}

The source maintains its own copy of the authenticated dictionary and provides the directory with the necessary information for updating. Such information contains the 
type of operation to be made, the element $(I d, C(I d))$, and a signed timestamp of the new value of the root node of $T$. When updating the dictionary, dynamic data structures $A_{1}, A_{2}$ and $T$ must be partially modified while maintaining the overall consistency of the system.

Updating $T$ consists of updating either $T_{1}$ or $T_{2}$ or both $T_{1}$ and $T_{2}$ and recomputing the root node of $T$. We suppose that $T_{2}$ is an "append/disjoin-only" Merkle tree, that is to say a Merkle tree in which incremental insertions/deletions are made on the right side of the tree.

Remark 2. The use of a static structure for $T_{2}$ allows us to obtain a proof (at least) as short as would be the case with a dynamic structure. Moreover, deletion and insertion remain efficient since the position of the elements does not depend on any rank.

Insertion of an Element. Insertion of a new pair element $(I d, c)$ where $H(I d) \notin$ $\left(u_{i}\right)_{i=1 \ldots n}$, is done in $T_{2}$ since we consider the data to have zero frequency (it has never been queried before). The following operations must be done on $T$.

- We first determine the largest index $j$ such that $u_{j}<H(I d)<u_{j+1}$.

- The existing leaf $h_{\Pi(j+1)}=H\left(u_{j}, u_{j+1}, c_{j+1}\right)$ is changed to $h_{\Pi(j+2)}=$ $H\left(H(I d), u_{j+1}, c_{j+1}\right)$.

- A new leaf $h_{\Pi(j+1)}=H\left(u_{j}, H(I d), c\right)$ is created on the right side of the tree.

- Internal nodes corresponding to paths from each of these two leaves to the root node of $T$ are recomputed.

Updating an Element. Here, we focus on the operation which changes the content of an existing element $(I d, c)$ to $c^{\prime}$.

- We find $j$ such that $u_{j}=H(I d)$.

- We set $h_{\Pi(j)}^{\prime}=H\left(u_{j-1}, u_{j}, c^{\prime}\right)$.

- We recompute the nodes of the path from the updated leaf $h_{\Pi(j)}^{\prime}$ to the root node (these nodes may belong to either $T_{1}$ or $T_{2}$ ).

Content Reordering. When the frequency of an element has changed, $T$ must be updated. There are three possibilities:

- The leaf belongs to $T_{1}$ and will stay in $T_{1}$.

- The leaf belongs to $T_{1}$ and will move to $T_{2}$.

- The leaf belongs to $T_{2}$ and will move to $T_{1}$.

In this paper, we focus on the first case, the two other cases are easier to deal with and are left to the reader. We describe an updating algorithm to maintain the frequencies in a certain descending order. For the sake of simplicity, we suppose that we just have to move one element of frequency $f_{m}(m>i)$ between $f_{i}$ et $f_{i+1}$. We avoid the use of cyclic permutations since it would lead to update too many nodes (the cost would be in $\mathrm{O}((m-i) \log (n)))$. We prefer to use an algorithm that we call min-max which limits to one the number of changes at each level of the tree $T_{1}$. Let $h$ be the depth of the tree. We denote by $S_{i}(i=1 \ldots h+1)$ the set of elements $(I d, C(I d))$ whose leaves 
belong to level $i$ of the tree. Let $f($.$) be the map which associates its frequency to a key$ identifier. At the same level, frequencies are not ordered but we must have:

$$
\forall i=1 \ldots h-1, \forall I d_{x} \in S_{i}, I d_{y} \in S_{i+1} f\left(I d_{x}\right) \geq f\left(I d_{y}\right) .
$$

Suppose that the leaf authenticating an element $e=(I d, C(I d))$ belonging to level $i$ must move up to level $j(i>j)$. This leaf is inserted at level $j$ at the position of the leaf having the lowest frequency in this level. This last element is moved down to level $j+1$ at the position of the leaf having the lowest frequency, and so on. The leaf having the lowest frequency at level $i-1$ is moved up to the former position of $e$ at level $i$. Finally, nodes which are on the path of the leaves that have been moved are recomputed back up the root of the tree. The algorithm is similar when $i<j$ except that the lowest frequency must be replaced by the highest frequency. If $i=j$, no change has to be done. If more than one frequency has changed, this algorithm can be applied for each change, albeit optimizations are possible but out of the scope of this paper.

Deletion of an Element. Here, we just outline the main steps of the deleting operation. Deleting an element leads to similar operations to that of reordering a leaf in $T$, with an updating of the pairwise chaining. Suppose that an element $e$ is deleted at level $i$, the highest frequency element at level $i+1$ is moved up to the position of $e$ and its position will be taken by the element having the highest frequency at the next level, and so on until level $h$. At level $h$, the element at the right side is moved to the position left empty. Suppose that the frequency of $e$ is $f_{i}$, then an updating of the pairwise chaining must be done. The new value of $h_{\left.\pi_{\left(\pi^{-1}\right.}(i)+1\right)}$ is $H\left(u_{\pi^{-1}(i)-1}, u_{\pi^{-1}(i)+1}, c_{\pi^{-1}(i)+1}\right)$. Furthermore, values of the ancestor nodes of leaves that have been moved have to be recomputed to restore the consistency of information.

\section{Complexity Analysis}

In this section, we analyze the complexity of the authentication part of our dictionary. We first concentrate on the size of a proof (of existence), then on the complexity of the proof construction and on the verification. Finally, we analyze the complexity of the updating operations. For the sake of simplicity, we express the operation cost in terms of the number of hash operations. We can then deduce the overall number of blocks processed by the hash function, which is approximately a multiple 1 of the number of hash evaluations.

Authentication Proof Size and Verification Run Time. In the following, we give the existence proof size in terms of the number of hash values needed to recompute the root node of $T$. The proof of non-existence for a hashed identifier, denoted $u^{*}$, is not detailed since this can be considered as a proof of existence for a particular hashed identifier $u_{i}$ such that $u_{i-1}<u^{*}<u_{i}$ for a given $i \in \llbracket 1, n+1 \rrbracket$. In this case the server has to provide to the client, in addition, the values of $u_{i}$ and $c_{i}$. We detail different cases, the best case, the worst case and the average case. From the average proof size standpoint,

\footnotetext{
${ }^{1}$ In our system, the number of blocks processed in one evaluation of the hash function can vary: this is the tree arity of $T_{1}$ plus one (that is, 4) for a node evaluation of $T_{1}$, the tree arity of $T_{2}$ (that is, 2) for a node evaluation of $T_{2}$ and 3 for a leaf evaluation.
} 
we discuss for which probability distribution it is preferable to use only $T_{1}$ or $T_{2}$, or the combination of both $(T)$. Note that we express the verification complexity in terms of number of hash operations. In our construction, this number is close to the number of hash values contained in the proof.

Theorem 1. Considering a number of elements $n \geq 1$, the authentication proof is of length 3 in the best case, and of length $\lceil\log (n-m)\rceil+2$ in the worst case, where $m$ is the Zipf distribution median.

Proof. In the best case, the requested content is the most frequently viewed. The leaf corresponding to the requested identifier is then located at the root node of $T_{1}$. Assuming that the requested identifier corresponds to a hashed value $u_{i}$, the user needs the preceding hashed identifier $u_{i-1}$, plus the hash of the concatenation of the sibling nodes $H\left(N_{2}, N_{3}\right)$ (Both $u_{i}$ and $c_{i}$ are determined locally, once the content is downloaded), and finally the root node value of $T_{2}$. In the worst case, the requested content is located in the tree $T_{2}$ which is an almost balanced binary tree, the user needs the preceding hashed identifier $u_{i-1}$ plus the siblings of the nodes along the path from the leaf to the root node, plus the root node value of $T_{1}$, for a total of at most $\left\lceil\log \left(N_{r}\right)\right\rceil+2$ values.

Average Case. If the queries are uniformly distributed in the set of elements, there is no reason to use the tree $T_{1}$, due to the overhead of the internal nodes. In this case we should only use the almost balanced binary tree $T_{2}$ in order to have an average size for the authentication proof tightly upperbounded by $\lceil\log (n)\rceil+1$. In order to show that the tree $T_{1}$ is useless in this case, let us determine the average proof size when we only use this tree. Consider $n$ such that $n=2^{m}-1$. The proof size for an internal leaf belonging to a level $i$, for $i \in \llbracket 1, m-1 \rrbracket$, is $2(i-1)+2$ hash values whereas it is $2(m-2)+2$ for a base level leaf (level $m$ ). Take the derivative of the geometric series $\sum_{i=0}^{m-1} x^{i+1}$ and simplify the following average proof size of $\frac{1}{\left(2^{m}-1\right)}\left(\sum_{i=0}^{m-2}(2 i+2) 2^{i}+2^{m-1}(2(m-\right.$ $2)+2)$ ). We then deduce that, when using only $T_{1}$, the average proof size is close to $2\lfloor\log (n)\rfloor$ hash values.

By contrast, when the queries are distributed according to a geometric distribution of parameter $p=1 / 2$ (which is close to a discrete equivalent of an exponential law), it is best to use only $T_{1}$. Indeed, by evaluating a geometric series, one can deduce that in such a case the average proof size is asymptotically 4 hash values.

As regards the Zipf distribution, it is preferable to use an authenticated data structure like $T$ because frequencies do not decrease as fast as an exponential law. Zipf is based on harmonic series and therefore it is difficult to provide a bound of complexity that closely reflects reality. Consequently, we give in Table 1 numerical results by varying the dictionary size, along with the percentage gain compared to what is obtained with the use of a standalone Merkle-like data structure.

Proof Construction. If, for each node of the tree, hashes of the concatenation of left and right children are stored in memory, the construction cost is equal to the cost of a searching operation (expressed as the number of comparisons) which is in $\mathrm{O}(\log (n))$. However, if these hashes have to be recalculated, the global cost is upper bounded by the number of hash calculations to be done on the path, which is itself upper bounded by the depth of the tree. 
Table 1. Average proof size and verification cost results

\begin{tabular}{||c|c|c|c||}
\hline Dictionary size & Merkle-like structure & Our system & Improvement \\
\hline \hline $10^{3}$ & 9.97 & 8.05 & $19.5 \%$ \\
\hline $5 \cdot 10^{4}$ & 15.61 & 12.25 & $22.5 \%$ \\
\hline $5 \cdot 10^{5}$ & 18.93 & 14.73 & $22.5 \%$ \\
\hline $10^{6}$ & 19.93 & 15.46 & $22.5 \%$ \\
\hline \hline
\end{tabular}

Update Complexity. We focus here on the updating of one element, in terms of hash computations.

Theorem 2. When modifying the content of an element, the number of hash evaluations to update $T$ is upper bounded by $\lceil\log (n)\rceil+2$ where $n$ is the overall number of elements in the dictionary.

Proof. We update a leaf for a cost of one hash evaluation. Ancestor nodes of this leaf until the root node of $T_{1}$ (or $T_{2}$ ) need to be updated for a cost bounded by $\lceil\log (n)\rceil$. Finally the root node of $T$ needs to be updated for a cost of one hash evaluation.

Theorem 3. When inserting a new element of frequency $f=0$, the number of hash evaluations is upper bounded by $2\lceil\log (n)\rceil+3$ where $n$ is the overall number of elements after insertion.

Proof. One pairwise link (one leaf) is changed in two pairwise links (2 leafs) for a cost of two hash function evaluations. The ancestor nodes of these two leaves, which can be located in $T_{2}$ or in $T_{1}$ and $T_{2}$, need to be computed (or re-computed) for an overall cost of at most $2\lceil\log (n)\rceil+2$ hash evaluations. Finally a last hash computation is needed to recompute the root node of $T$.

Theorem 4. Assume that the absolute frequency of one element has changed and that this element which belongs to $T_{1}$ stays in $T_{1}$, the number of hash computations needed to meet the order property (1) is at most $\frac{\lfloor\log (n)\rfloor(\lfloor\log (n)\rfloor+1)}{2}$.

Proof. Let us suppose that the frequencies of the leaves from the root to the base level and from left to right are denoted $f_{1}, f_{2}, \ldots, f_{n}$. We consider the worst case which appears when a leaf of the base level needs to be moved at the root level, for instance if the frequency $f_{n}$ is changed in $f_{n}^{*}$ and $f_{n}^{*}>f_{1}$. Let $h$ be the depth of the tree. In this scenario, by using the "min-max" choice criteria, we move the leaf $h_{n}$ to the root level, while $h_{1}$ is moved down to the next level at the position of the leaf of lowest frequency. This last leaf is itself moved to the next level at the position of the leaf of lowest frequency, and so on, until the base level is reached. The lowest frequency leaf at level $h$ is moved to the former position of $h_{n}$ at level $h+1$ (the base level). Overall, one leaf has been replaced at each level of the tree. Nodes along the path from changed nodes to the root node are updated. This non-optimal strategy leads to the following upper bound on the number of hash computations: $\sum_{i=1}^{h} i=\frac{\lfloor\log (n)\rfloor(\lfloor\log (n)\rfloor+1)}{2}$ 
Theorem 5. When deleting an element in $T_{1}$, by using the "min-max" choice criteria, the number of hash computations is in $\mathrm{O}\left(\log (n)^{2}\right)$.

Proof. Operations are similar to that of reordering an element, except that one leaf and its ancestor nodes need to be recomputed.

Search Complexity. The cost of a search operation is given in terms of comparisons.

Search of a content: Since non authentifying structures use well known mechanisms, we do not describe the search algorithm. The content and frequency associated to a given $u_{i}$ is obtained using $A_{1}$ and is done in $\mathrm{O}(\log (n))$ comparisons. The rank of the frequency is obtained using $A_{2}$ and is also done in $\mathrm{O}(\log (n))$ comparisons. Globally, the search of a leaf and the construction of the proof is done in $\mathrm{O}(\log (n))$ comparisons.

Search of an element: The cost is done in $\mathrm{O}(\log (n))$ comparisons for insertion and modification of an element. The position of a leaf having a minimal (or maximal) frequency in a given level of the tree is done in $\mathrm{O}(\log (n))$ comparisons.

Remark 3. From the previous analysis, we can deduce that reordering or deleting operations is done in $\mathrm{O}\left(\log (n)^{2}\right)$ comparisons.

\section{The Choice of the Structure}

Our objective is to optimize the average proof size, avoiding the expensive worst cases. With our structure, the maximal proof length is bounded by $\lceil\log (n)\rceil+2$. A dynamic Huffman tree gives slightly better results for the average case but the proof size in the worst case is in $\mathrm{O}(n)$ for outlier (discrepant) samples. This worst case occurs for example when the distribution is exponential. An alternative is to use a dynamic Huffman tree for a small subset of data. This solution limits the expensive cost of the worst case. When considering a sample distant from Zipf's law, we may obtain a degenerate tree. In that case, the use of a length-limited Huffman tree [11] may be considered. Note that our structure has the advantage of simplicity (in particular, the construction of our tree is done in $\mathrm{O}(n)$ ). Moreover, it provides all needed operations while keeping append/disjoin-only structures.

\section{A Framework to Authenticate Http Responses}

The aforementioned authenticated dictionary can be used for authentication of HTTP responses of a Web server [2] when the request distribution follows Zipf's law. The server returns either the requested page together with a 200 success response or a 404 error message and a proof of authenticity of the content of that page (or possibly a proof of non existence). In this context, $u_{i}$ represents the hash of a url (Uniform Resource Locator) and $c_{i}$ is the content of the corresponding page. However, it is important to note that in a dynamic site, a page has many contents which vary over time. Hence, it makes no sense to consider the hash of a page. When creating a page, one should define a scheme allowing authentication to be performed on the static fields which are of interest to the user. 


\section{Conclusion}

We have proposed a model for authenticated dictionaries which takes into account the frequency of queries with the aim of obtaining a smaller proof size. This contrasts with the assumption made by existing dictionaries that the frequency distribution is uniform. Based on a frequency distribution following Zipf's law, we introduced a data structure with two components, each of which being nearly optimal for a portion of the distribution. We obtained an average gain of more than $20 \%$ on proof size while response time remains similar. In our complexity analysis, comparisons were made with a Merkle tree which is less costly than the dynamic structures used in [8]. However, since our system provides operations like insertion and deletion, it could also be compared to these dynamic structures, with the expectation that even greater gains would be realized.

In this paper, we have not discussed possible optimizations, including the use of length-limited Huffman trees, which will be developed in future papers.

\section{References}

1. Adamic, L.A., Huberman, B.A.: Zipf's law and the internet. Glottometrics 3, 143-150 (2002)

2. Bayardo, R.J.: Merkle tree authentication of http responses. In: In Proc. 14th WWW, pp. 1182-1183 (2005)

3. Blibech, K., Gabillon, A.: Chronos: an authenticated dictionary based on skip lists for timestamping systems. In: SWS, pp. 84-90 (2005)

4. Buldas, A., Laud, P., Lipmaa, H.: Accountable certificate management using undeniable attestations. IACR Cryptology ePrint Archive, 2000:27 (2000)

5. Devanbu, P., Gertz, M., Martel, C., Stubblebine, S.G.: Authentic Third-party Data Publication. In: Thuraisingham, B., van de Riet, R., Dittrich, K.R., Tari, Z. (eds.) 14th IFIP 11.3 Working Conference in Database Security. IFIP, vol. 73, pp. 101-112. Springer, Heidelberg (2000)

6. Easley, D., Kleinberg, J.: Power laws and rich-get-richer phenomena. In: Networks, Crowds, and Markets: Reasoning about a Highly Connected World. Cambridge University Press (2010)

7. Gassko, I., Gemmell, P.S., MacKenzie, P.D.: Efficient and fresh certification. In: Imai, H., Zheng, Y. (eds.) PKC 2000. LNCS, vol. 1751, pp. 342-353. Springer, Heidelberg (2000)

8. Goodrich, M.T., Tamassia, R.: Efficient authenticated dictionaries with skip lists and commutative hashing. Technical report, Johns Hopkins Information Security Institute (2001)

9. Goodrich, M.T., Tamassia, R., Schwerin, A.: Implementation of an authenticated dictionary with skip lists and commutative hashing 2, 68-82 (2001)

10. Kaufman, C., Perlman, R.J., Speciner, M.: Network security - private communication in a public world. Prentice Hall series in computer networking and distributed systems. Prentice Hall (1995)

11. Larmore, L.L., Hirschberg, D.S.: A fast algorithm for optimal length-limited huffman codes. J. ACM 37(3), 464-473 (1990)

12. Mahanti, A., Carlsson, N., Mahanti, A., Arlitt, M., Williamson, C.: A tale of the tails: Powerlaws in internet measurements. IEEE Network 27(1), 59-64 (2013)

13. Mehta, D.P., Sahni, S. (eds.): Handbook of data structures and applications. Chapman \& Hall/CRC (2005)

14. Merkle, R.: Protocols for public key cryptosystems. In: SIMMONS: Secure Communications and Asymmetric Cryptosystems (1982) 
15. Merkle, R.C.: A certified digital signature. In: Brassard, G. (ed.) CRYPTO 1989. LNCS, vol. 435, pp. 218-238. Springer, Heidelberg (1990)

16. Naor, M., Nissim, K.: Certificate revocation and certificate update. IEEE Journal on Selected Areas in Communications 18(4), 561-570 (2000)

17. Nielsen, J.: Do websites have increasing returns (1997), http://www.nngroup.com/ articles/do-websites-have-increasing-returns/

18. Nielsen, J.: A note about page popularity (2006), http://www.nngroup.com/articles/traffic-log-patterns/

19. Preneel, B., Van Rompay, B., Quisquater, J.J., Massiasand, H., Serret Avila, J.: Design of a Timestamping System (1999)

20. Pugh, W.: Skip lists: A probabilistic alternative to balanced trees (1990)

21. Saichev, A.I., Malevergne, Y., Sornette, D.: Theory of Zipf's Law and Beyond. Lecture Notes in Economics and Mathematical Systems. Springer (2009)

22. Tamassia, R.: Authenticated data structures. In: Di Battista, G., Zwick, U. (eds.) ESA 2003. LNCS, vol. 2832, pp. 2-5. Springer, Heidelberg (2003) 